\title{
Análisis retrospectivo en el estudio epistemológico de conceptos y objetos matemáticos: una propuesta metodológica para lograr la metacognición
}

\author{
Retrospective analysis in the epistemological study of concepts and objects: \\ a methodological proposal to achieve metacognition
}

Winston Joseph Zamora Díaz

\section{Resumen}

Este articulo ha descrito una propuesta metodológica para lograr la metacognición. Se trata de un esbozo argumentativo que analiza de forma retrospectivo en el estudio epistemológico de conceptos y objetos matemáticos. En fin, el propósito de este artículo no es proveer un recorrido histórico sobre el surgimiento y evolución de la Matemática Educativa, pero si destacar que, por su reciente evolución, es urgente y pertinente generar propuestas o metodologías que orienten hacia la investigación matemática como recurso que afiance mejores alternativas para la enseñanza y aprendizaje de las matemáticas, principalmente cuando nos referimos a la formación de profesores de matemática. Es por tal razón que el contenido medular del artículo es presentar algunas ideas acerca del análisis temporal para el estudio epistemológico de conceptos y objetos matemáticos y potenciar la metacognición. Esta iniciativa se propone a manera de una metodología que permita gestionar y desarrollar la investigación matemática en las escuelas universitarias donde se forman profesores de matemática.

Palabras clave: Metacognición, objetos matemáticos, conceptos matemáticos, epistemología, didáctica de las matemáticas.

\section{Abstract}

This article has described a methodological proposal to achieve metacognition. It is an argumentative sketch that analyzes retrospectively in the epistemological study of mathematical concepts and objects. Finally, the purpose of this article is not to provide a historical overview on the emergence and evolution of Educational Mathematics, but if we highlight that, due to its recent evolution, it is urgent and pertinent to generate proposals or methodologies that guide mathematical research as a resource that assists better alternatives for the teaching and learning of mathematics, especially when we refer to the training of mathematics teachers. It is for this reason that the core content of the article is to present some ideas about the temporal analysis for the

1 Doctor en Educación e Intervención Social. Director Académico de la Universidad Panamericana de Nicaragua. Email: winzamora@ yahoo.com (10: https://orcid.org/oooo-0003-2734-3364

Recibido: 03/03/2019 Aprobado: 05/05/2019

Zamora-Díaz, W. (2019). Análisis retrospectivo en el estudio epistemológico de conceptos y objetos matemáticos: una propuesta metodológica para lograr la metacognición. Revista Electrónica de Conocimientos, Saberes y Prácticas, 2(1), 76-86. D0I: https://doi.org/10.5377/recsp. v2i1.8168 
epistemological study of mathematical concepts and objects and to promote metacognition. This initiative is proposed as a methodology for managing and developing mathematical research in university schools where Mathematics teachers are trained.

Keywords: Metacognition, mathematical objects, mathematical concepts, epistemology, didactics of mathematics

\section{Introducción}

Desde que surge la enseñanza y aprendizaje de las matemáticas, el cómo mejorar este proceso, ha sido una aspiración de manera incesante por parte de los que nos ocupamos de la actividad docente. Todos los profesores de matemática destacamos que la Matemática constituye una forma de aproximación a la realidad; brinda elementos de gran importancia para el desarrollo de la capacidad de argumentación racional, la abstracción reflexiva, la resolución de problemas no sólo de índole escolar, sino de gran aplicación y transferencia en otros campos del saber (Castro, 2007). Sin embargo, la inquietud de cómo contribuir a mejorar el proceso de aprendizaje de esta área del conocimiento aun continúa siendo un gran dilema. Para ello a continuación detallamos algunas evidencias (a manera de pinceladas) que ponen de manifiesto cómo se ha venido presentando el problema.

De forma general me atrevo a decir que la mayor parte de las generaciones anteriores de matemáticos, aunque vastos de mucho conocimiento, se dedicaron en su mayoría a transcribir el conocimiento matemático, tal y como ellos lo comprendían; sin importar realmente de qué manera lo concebirían los estudiantes. Para los años 50 (1952) del siglo pasado comenzó a tener auge un numeroso grupo de profesores de matemática en Estados Unidos de Norteamérica con lo que surge la Educación Matemática o Matemática Educativa (desde el término anglosajón), este grupo atendía de forma preocupada un cambio radical en el cómo enseñar matemáticas y qué matemáticas enseñar, se presentan propuestas desde la didáctica de la matemática, objeciones a los planes de estudio y debates sobre el abordaje de distintos tópicos de matemática general y la matemática superior.

La siguiente pregunta y comentario estuvieron presentes en este grupo en 1952:

“QQué valor educativo puede tener un estudio de los exponentes, los radicales, los logaritmos, la regla de Horner, las fracciones simples, el teorema del binomio de Newton, las identidades trigonométricas, etc.?, pues se oye decir que los estudiantes aprenden a pensar al estudiar esas cosas. Sin embargo, el autor afirma que la materia no se presta a ello. "Todos sabemos que es imposible dar a un freshman (es como un estudiante primer año de secundaria) una definición satisfactoria de número irracional. 


\section{REFLEXIONES EDUCATIVAS}

Del mismo modo estos profesores discutían que cómo era posible continuar enseñando demostraciones con argumentos intuitivos, evidencias gráficas y afirmaciones aceptadas sin pruebas. "Aún así esperamos que el alumno lo aprenda todo bien".

Aunque las preocupaciones por la enseñanza de las matemáticas y su mejora progresiva se muestran tan antiguas como la enseñanza de esta, el estudio sistemático por atender a este fenómeno en América Latina es reciente (Cantoral y Farfán, 2002). Así en México la Sección de Matemática Educativa surge a partir de 1975 en el Centro de Investigación y de Estudios Avanzados (Cinvestav) del Instituto Politécnico Nacional (IPN). Desde entonces este grupo de investigadores dedicados al desarrollo de este centro de investigación han destinado metodologías y recursos a la formación de nuevos profesionales de la Matemática Educativa. Sin embargo, a pesar de ello, sigue habiendo mucho insumo por investigar y consolidar en términos de qué matemática enseñar, para que enseñar lo que decimos que es necesario, y cómo enseñarlo.

De manera análoga en Colombia en la década de los ochenta, después de haber enfrentado un "Caos Curricular" 2 como lo llamaron algunos, surge la necesidad de hacer una profunda reflexión epistemológica acerca del quehacer de la enseñanza de la matemática. Entre las estrategias citadas en las políticas colombianas estuvo la fortalecer las competencias del profesorado de matemáticas. Y para 1991 una nueva ley propone una evaluación por logros y los procesos curriculares se enfocan hacia una consecución de habilidades cognitivas y cognoscitivas que dieran indicativos del dominio de competencias. Los dedicados a la investigación del proceso de enseñanza y aprendizaje en Colombia siguen buscando propuestas pedagógicas y metodológicas que propicien el pensamiento matemático correctamente estructurado.

Con el mismo deseo de superar esta inquietante cuestión Costa Rica, a pesar de destacar entre sus indicadores educativos, y de poseer universidades que posicionan entre las mejores de América Latina como la Universidad de Costa Rica (UCR), para el año 2017 muestra preocupación por desarrollar la didáctica de las matemáticas en la formación de profesores de matemática y para ello da apertura a la carrera de Educación Matemática, potenciando de esta forma las didácticas de las distintas ramas de la matemática.

En Nicaragua, en lo que a desarrollo curricular se refiere en el área de las matemáticas, en las últimas dos décadas el Ministerio de Educación se ha enfocado en el transferir modelos de otros contextos socioeconómicos, entre ellos el Constructivismo, Enfoque para la Comprensión (EPC), el enfoque por competencias, enfoque resolución de problemas. Todo esto en el marco del apoyo de la cooperación internacional, entre ellas la que mayor sobresale es JICA que es un fondo de cooperación japonesa. Sin

2 Se dio con el surgimiento de la ley 115, "Ley General de Educación”, cuando el Ministerio de Educación Nacional deja de regular el currículo escolar y confiere a cada institución desarrollar su propio proyecto. 
embargo, los resultados catastróficos a la hora de develar los resultados de evaluaciones de las habilidades matemáticas en los exámenes de ingreso a las universidades continúan en espera de una receta mágica. Actualmente a pesar de los esfuerzos del gobierno por invertir en el acceso a bibliografía en las escuelas, a proveerle a los maestros guías metodológicas, sigue estando vigente la necesidad de trabajar e investigar por propuestas de desarrollo o cambios de paradigmas (Lucio, 2015) para una mejor enseñanza de esta asignatura, y de fortalecer además los planes curriculares de los profesores que se forman en las universidades.

En fin, el propósito de este artículo no es proveer un recorrido histórico sobre el surgimiento y evolución de la Matemática Educativa, pero si destacar que, por su reciente evolución, es urgente y pertinente generar propuestas o metodologías que orienten hacia la investigación matemática como recurso que afiance mejores alternativas para la enseñanza y aprendizaje de las matemáticas, principalmente cuando nos referimos a la formación de profesores de matemática. Es por tal razón que el contenido medular del artículo es presentar algunas ideas acerca del análisis temporal para el estudio epistemológico de conceptos y objetos matemáticos y potenciar la metacognición. Esta iniciativa se propone a manera de una metodología que permita gestionar y desarrollar la investigación matemática en las escuelas universitarias donde se forman profesores de matemática.

\section{Cuestiones generadoras de investigación y reflexión}

Como en todo proceso reflexivo- investigativo, independientemente del método a seguir es necesario convenir un hilo conductor que justifique la ruta de indagación. En este caso por tratarse de un artículo, primero de revisión bibliográfica, se ha acudido a hacer referencias de aquellos autores y teorías que consideramos pertinentes para dar contenido a la temática. Y segundo por tratarse de una alternativa teórica para aplicarla en la formación de profesores de matemáticas y para fomentar la investigación en este campo; es que se procura detallar aspectos considerados claves que dan contenido a esta iniciativa.

A continuación, mencionamos algunas cuestiones que incitaron al desarrollo:

- "El 8o\% de lo que se aprende en la asignatura de matemáticas no sirve para nada”. Conrad Wolfran, Físico y Matemático Inglés.

- Además de incluir sistemáticamente una reflexión acerca de la enseñanza de algunos estándares en cada nivel,... las sugerencias para implementar su enseñanza son muy prácticas. Se basan en investigaciones y referencias constantes a experiencias de aula que muestran cómo hacer matemáticas en aula. (NCTM, 2003).

- ¿Qué matemáticas debemos enseñar? 


\section{REFLEXIONES EDUCATIVAS}

- Enseñar y aprender matemáticas desde un nuevo paradigma (Lucio, 2015). ¿Cuál (es)?

- ¿Qué es lo que esperamos con lo que enseñamos de matemática?

- ¿Qué caminos recorre o debe recorrer la construcción del conocimiento matemático? (referencia a la epistemología de la matemática).

- ¿Qué conozco de este tema?, ¿tengo claro el significado de...?, ¿Qué conclusiones puedo sacar? (metacognición).

- ¿Qué metodologías se pueden utilizar para hacer más favorable el proceso de enseñanza y aprendizaje de las matemáticas, y que las mismas recuperen el sentido de estar presentes en la escuela?

\section{Desarrollo de elementos que sustentan esta propuesta teórica}

Siendo la educación una actividad de ocurrencia social, claro está que para entenderla y modificarla como proceso, es necesario compartir significados. Por tanto, no basta con medirla, ni documentar el proceso, es necesario procurar entender las distintas realidades y problemáticas presentes en la enseñanza y aprendizaje.

Cabe resaltar que es frecuente que los responsables a cargo de la organización curricular de los contenidos a tratar en las escuelas, escazas veces lo hacen de forma integrada, como que el currículo pudiera ser un objeto de estudio por separado. De acuerdo con Chrobak (2000), es pertinente señalar que las consideraciones epistemológicas son un factor de gran ayuda para interpretar tanto el currículo como la generación de conocimientos y por tanto el aprendizaje.

Siendo la Universidad una institución comprometida con la formación de profesores de matemáticas debe alertar y promover la necesidad de revisar lo que se ha venido haciendo, y cómo hacerlo cada vez mejor. Por lo tanto, será también responsabilidad de ésta poner a la disposición diversas perspectivas teóricas en función de la praxis docente, la evolución del conocimiento y la investigación en el área de matemática (Flores, Araya y Zamora, 2017).

A pesar de los majestuosos esfuerzos investigativos que han desarrollado muchas universidades, los cambios acerca de la concepción de la enseñanza y aprendizaje de esta ciencia siguen siendo minúsculos. No es posible reducir esta problemática actual a recetas mágicas, ni a simples prácticas manipulables de campo, ni al abuso de la lúdica. Lo prometedor sería la revisión compartida de los sujetos involucrados, la reflexión y por supuesto el estudio de prácticas de aula desde la investigación -acción y la sistematización. Estamos convencidos, que debemos penetrarnos en dos concepciones de mucha pertinencia para una profunda reflexión. Una de ellas es la de la didáctica de las matemáticas no sólo como el arte de enseñar, sino como la ciencia del desarrollo de las planificaciones realizables en la enseñanza de la matemática; y 
como la disciplina encargada de estudiar y comunicar de forma crítica, propuestas y actuaciones que impliquen el rol docente y de los estudiantes.

Y la otra es la concepción epistemológica de las matemáticas, como el conjunto de convicciones, de conocimientos y de saberes científicos que tienden a decir cuáles son los conocimientos de los individuos o de los grupos de personas, su funcionamiento, y las formas de establecer su validez, de adquirirlas y por tanto de aprenderlas y enseñarlas. "La epistemología es un tentativo de identificar y de unificar diversas concepciones" (Brousseau, 2006).

\subsection{La epistemología en la generación del conocimiento}

Desde las épocas de Platón, la filosofía y el conocimiento nacen y evolucionan con el aporte de diversos pensamientos e ideas de otros. Y es así como la filosofía, como madre de todas las ciencias, se fortalece con la aparición del logos, y así comienzan las preguntas acerca del origen de todo.

En este apartado hacemos hincapié en la epistemología puesto que no debemos perder de vista que es la lógica del conocimiento, y por tanto la rama de la filosofía que a través de la investigación científica y distintas metodologías permite generar el conocimiento. También se le ha denominado filosofía de la ciencia, y tiene por objetivo conocer las cosas en su esencia y en sus causas; la epistemología se ocupa de los enunciados de la ciencia y de sus relaciones lógicas, la validez del conocimiento y, lo que, es más, las condiciones de acceso al conocimiento válido (González, 2013).

Para aproximarnos un poco a la epistemología como un proceso en la generación del conocimiento, en este caso particular a la del conocimiento matemático, deseo remarcar primero la definición presentada por el Dr. Cantoral acerca de lo que es la Matemática Educativa. El mismo lo define así:

La matemática educativa, tiene una historia particular estrechamente vinculada con una comunidad de investigadores que se planteó el problema de estructurar la matemática con fines educativos. La matemática educativa tiene un pequeño matiz que la distingue de otras visiones. Voy a intentar ejemplificarlo. No nos interesa solamente cómo hacer que alguien aprenda. Nos interesa también entender cómo tendría que construirse el conocimiento si el fin es su aprendizaje..., así para nosotros la matemática escolar es algo que se construye, no hay contenidos fijos ni formulaciones únicas (Cantoral, 2000).

Esta concepción nos induce a preguntarnos ¿cómo se construye el conocimiento matemático?, se trata de ir más allá de la tradicional transposición que transfiere el conocimiento del erudito a la pizarra, y centrarnos en función de cuál es/fue la lógica de ese saber para que sea hoy un conocimiento acabado. Nos interesa reconocer 


\section{REFLEXIONES EDUCATIVAS}

¿Cuáles son los orígenes del conocimiento científico?, de qué manera se transforma el tratamiento de un objeto matemático de un investigador a otro, de un país a otro, de una institución educativa a otra, con qué fines o intereses y sostenidos en qué lógica.

Los educadores e investigadores matemáticos debemos procurar una epistemología de la matemática que se interese más en estudiar los fundamentos de validez de los conceptos u objetos matemáticos, así también las condiciones y contextos de descubrimiento pasados y presentes. No todos los educadores matemáticos comparten la misma epistemología, incluso aunque se interesen con cuestiones epistemológicas similares (Sierpinska \& Lerman, 1996).

\subsection{Algunas mociones sobre el análisis temporal: enfoque retrospectivo}

De manera tradicional hemos venido desarrollando el proceso de enseñanza de las matemáticas enterando al estudiante del conocimiento acabado al que tenemos alcance. Pareciera como que no nos planteáramos dudas o la necesidad de profundizar en dichos conocimientos; o más bien cómo si no importara del todo el proceso de evolución de este y la lógica del cómo estudiarlo para comprenderlo. Dentro del proceso de gestión, consolidación y evolución del conocimiento matemático susceptible de ser estudiado, es inexcusable el desarrollo de un proceso de investigación. Éste nos remonta a la explicación del origen, de la obtención del conocimiento, y la predicción de sus aplicaciones en el comportamiento de un fenómeno o en la solución de un problema.

Hay conceptos u objetos matemáticos que durante se comunican y aprenden requieren de los distintos enfoques con los que suceden los procesos de investigación. Nos referimos al nivel de profundidad con que se desee abordar un contenido, estos pueden ser descriptivos, explicativos y predictivos. En consecuencia, con esto, cabe destacar que para conocer a profundidad y poder ampliar explicaciones sobre un determinado conocimiento matemático, se ha estado obviando la necesidad de incluir en el estudio de éste una alternativa que nos remonte a ¿Qué pasó para surgiera este conocimiento?, ¿Por qué pasó? Las interrogantes planteadas nos conducen a buscar respuestas de acuerdo con el momento en el que ocurrió el conocimiento y al registro de este. A eso los expertos en métodos de investigación denominan análisis retrospectivo (Müggenburg y Pérez, 2007). Todo esto sin desligarlo del análisis circunspectivo que nos permitiría contestarnos ¿Qué está pasando en este momento con ese conocimiento?, ¿cómo se está tratando o con qué enfoque se aborda ese objeto matemático?, es posible que el segundo análisis provoque al primero o recíprocamente.

El análisis retrospectivo es un procedimiento que permite mirar hacia atrás. Un enfoque descriptivo que revisa acciones o hechos pasados con el objetivo de llegar a conclusiones veraces que ayudan a entender el porqué de algunas situaciones que reconocemos y damos sentido en el presente. Los antecedentes de una temática podrían hacer posible meta concebir un concepto u objeto de estudio. Además, desde esta 
opción podrían llegar a encontrarse errores cometidos, enunciar otras alternativas demostrativas de dicho objeto, hasta proveer nuevas rutas de indagación conceptual y procedimental para la evolución de este conocimiento. Por otro lado, este tipo de propuesta metódica de desarrollo del conocimiento matemático nos permitiría también ahondar el aspecto cultural y social del aprendizaje desde distintas sociedades o contextos en los que surge y se desarrolla el conocimiento.

Aunque este enfoque surgió en los años 50 en el campo de ciencia médica, por su perspectiva, podría ser puesta en práctica para el estudio y desarrollo de otras áreas del conocimiento, de manera particular en la ciencia matemática

\subsection{La metacognición y la articulación de conocimiento}

En este artículo se ha retomado aquella postura, que describe la preocupación de muchos maestros de matemáticas por lograr en sus estudiantes lo que se pregona como aprendizajes significativos. Sin embargo, comúnmente el proceso (enseñanza y aprendizaje) dirigido desde las escuelas en la mayoría de nuestras instituciones educativas niega esta perspectiva.

Si concebimos la metacognición como una alternativa viable para formar alumnos autónomos, sobre la base de una educación que potencie la conciencia sobre los propios procesos cognitivos y la autorregulación de los mismos por parte de los estudiantes, de tal manera que les conduzca a un "aprender a aprender", y a autodirigir su aprendizaje y transferirlo a otros ámbitos de su vida (Osses y Jaramillo, 2008), entonces nos veríamos obligados a reestructurar nuestras prácticas docentes, la formación de profesores de matemáticas y algunas prácticas institucionales que moderan los procesos de enseñanza y aprendizaje; entre otras cuestiones. Es real reconocer que las matemáticas en la escuela han perdido sentido y por tanto interés por ser estudiadas, es por tanto prioritario tomar acciones que hagan concretar las siguientes concepciones de metacognición.

La primera como la dimensión del conocimiento más abstracta que lleva al conocimiento de la propia persona (Jasso, 2014). Y la segunda Porque la enseñanza no se refiere a la transferencia de conocimiento, sino a la creación de posibilidades de su producción o de su construcción (Freire, 2012). Ante estas desafiantes concepciones nos queda hacernos las siguientes interrogantes: ¿qué hacemos desde nuestra concepción de trabajo docente?, ¿cómo concebimos el proceso de planificación o estructuración de nuestras clases?, ¿realmente me es posible orientar a mis estudiantes hacia el descubrimiento?, ¿cómo concibo la motivación para despertar el interés por el estudio de las matemáticas?, ¿cómo incito hacia la regulación de los aprendizajes a mis estudiantes?; estas son sólo algunas de las preguntas que deberíamos hacernos día a día, en cada momento de nuestro quehacer como docentes. Y repensar así 


\section{REFLEXIONES EDUCATIVAS}

cómo podemos contribuir desde nuestra formación continua, desde la reflexión y la investigación permanente.

El paso que deseamos alcanzar es el de erradicar la realidad de que siendo matemático, uno no pueda de ninguna manera garantizar el valor, menos la validez, del empleo de la matemática en un campo que es ajeno a uno mismo, puesto que es ajeno a la matemática;...y entonces semejante actitud conduce a interpretar los saberes como obras puras, obras sin objeto, sin razones de ser, como órganos sin funciones; como simples monumentos que uno se conforma con visitar (Chevallard, 2013).

Se requiere pues de una apertura epistemológica que permita la metacognición de los saberes matemáticos, que invaliden el horror instrumental que encierra el pensamiento de los más anti utilitaristas, y supere el enfoque monumental de los saberes. Y me parece que de esa manera será posible responder, desde la metacognición. ¿Cómo hacer esto? ¿Qué podría producirse si...?, y es que los saberes como lo expresa Chavellard (2013) aportan medios de actuar y de comprender..., son una fuente de inteligencia de las situaciones del mundo y de potencia en la acción dentro de esas situaciones (pp.19-20).

\section{Conclusiones y perspectivas futuras}

De todo lo expresado deseamos incitar hacia la gestión y reconstrucción del conocimiento, a partir de los antecedentes implicados en su construcción, o por qué no a partir de lo que Brousseau (1988a) llamaba la construcción de una situación fundamental, pero además se debe pretender sistematización y evolución de este. Por eso debemos ocuparnos de cómo el estudiante ha adquirido ese conocimiento, bajo que premisas y nociones previas, y a la vez cómo organizarlo para que el aprendizaje sea validado, perdure, lo comunique, y lo aplique en diferentes contextos y situaciones.

Se deben propiciar y evidenciar cambios en los procesos de enseñanza y aprendizaje de las matemáticas, todo esto desde enfoques que promuevan la apertura a la epistemología y a la didáctica. Incitar desde conversaciones y redes de trabajo una práctica más articulada (distintos subsistemas de educación) que promueva una enseñanza de las matemáticas que permita reconocer el sentido que se discursa acerca de la importancia de estudiar matemáticas. Fortalecer la visión formadora del profesorado de matemáticas por comprender que es necesario ahondar en la epistemología de la matemática, sin descuidar los distintos ritmos de aprendizajes, la gestión del conocimiento, la investigación- acción como fuente de reflexión de nuestras prácticas docentes.

Garantizar que la metacognición sea una dimensión presente en nuestras propuestas curriculares y en nuestra práctica docente permanente. Esto desde la perspectiva 
de que las actividades permitan una reflexión del conocimiento y los aprendizajes la muestra de capacidades de nuestros estudiantes.

\section{Lista de referencias}

Brousseau, G. (1988a). Le Contrat didactique: le milieu. Recherches en Didactique des mathématiques. Vol 9/3, 309-336. La pensé Sauvage, Grenoble.

Brousseau, G. (2006). Theory of didactical situations in mathematics: Didactique des mathématiques, 1970-1990 (Vol. 19). Springer Science \& Business Media.

Cantoral, R. (2002). La entrevista fue realizada en el marco de la decimocuarta Reunión Latinoamericana de Matemática Educativa (Relme 14) en Panamá, en julio de 2000.

Castro, J. (2007). La investigación en educación matemática: una hipótesis de trabajo. Revista Educere: Investigación Arbitrada, Año 11, No. 38. Pág. 549.

Chevallard, Y. (2013). La matemática en la escuela. Por una revolución epistemológica y didáctica. Buenos Aires. libros del Zorzal.

Chrobak, R. (2000). La metacognición y las herramientas didácticas. Universidad Nacional del Comahue. Facultad de Ingeniería, Departamento de Física. Buenos Aires. Recuperado el, 15.

Flores, J., Araya, A., Zamora, W. (2017). Perspectivas didácticas para la enseñanza y aprendizaje de las matemáticas: Una propuesta para mejorar el micro-planificación metodológico y temático. Managua: Sociedad de Jóvenes Escritores.

Freire, P. (2012). Pedagogía de la autonomía. Saberes necesarios para la práctica educativa. Siglo XXI. México.

Gil, R. (2015). "Enseñar y aprender matemáticas desde un nuevo paradigma”. El Nuevo Diario. 01 de noviembre 2015. Consultado en:

González, N. (2013). La epistemología de las matemáticas y su contribución al aprendizaje significativo en estudiantes de grado sexto. Tesis de Maestría en Enseñanza de las Ciencias Exactas y Naturales. Universidad Nacional de Colombia.

Jasso, J. (2014). Estrategias metacognitivas para articular el conocimiento, México. Universidad de Monterrey.

Marín, A y Lupiáñez, J. (2005). PRINCIPIOS Y ESTÁNDARES PARA LA EDUCACIÓN MATEMÁTICA. Revista Suma. SAEM Thales, Universidad de Granada. 
Müggenburg, M. y Pérez, I. (2007). Tipos de estudio en el enfoque de investigación cuantitativa. Revista Enfermería Universitaria. Vol 4. No. 1.

Osses, S. y Jaramillo, S. (2008). Metacognición: un camino para aprender a aprender. Estudios Pedagógicos XXXIV, 34(1), 187-197.

Ponteville, C. (200o). Acerca de la Matemática Educativa: Entrevista realizada

Sierpinska, A. y Lerman, S. (1996). Epistemologies of mathematics and of mathematics education. En: A. J. Bishop et al. (eds.), International Handbook of Mathematics Education (827-876). Traducción parcial de Juan Godino. Consultado en: http:// www.ugr.es/ jgodino/siidm/escorial/SIERLERM.html 